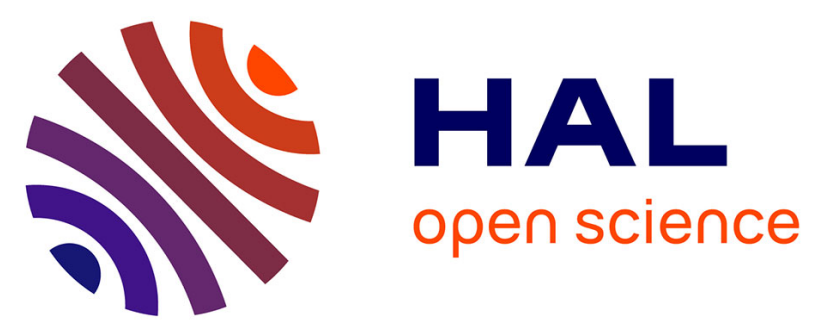

\title{
Experimental characterization and constitutive modeling of the biomechanical behavior of male human urethral tissues validated by histological observations
}

Christopher Masri, Grégory Chagnon, Denis Favier, Hervé Sartelet, Edouard Girard

\section{To cite this version:}

Christopher Masri, Grégory Chagnon, Denis Favier, Hervé Sartelet, Edouard Girard. Experimental characterization and constitutive modeling of the biomechanical behavior of male human urethral tissues validated by histological observations. Biomechanics and Modeling in Mechanobiology, 2018, 10.1007/s10237-018-1003-1 . hal-01807582

\section{HAL Id: hal-01807582 \\ https://hal.science/hal-01807582}

Submitted on 13 Jul 2018

HAL is a multi-disciplinary open access archive for the deposit and dissemination of scientific research documents, whether they are published or not. The documents may come from teaching and research institutions in France or abroad, or from public or private research centers.
L'archive ouverte pluridisciplinaire HAL, est destinée au dépôt et à la diffusion de documents scientifiques de niveau recherche, publiés ou non, émanant des établissements d'enseignement et de recherche français ou étrangers, des laboratoires publics ou privés. 


\title{
Experimental characterization and constitutive modeling of the biomechanical behavior of male human urethral tissues validated by histological observations
}

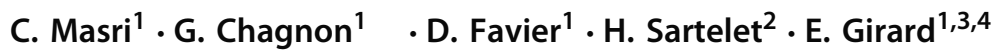

\begin{abstract}
This work aims at observing the mechanical behavior of the membranous and spongy portions of urethrae sampled on male cadavers in compliance with French regulations on postmortem testing, in accordance with the Scientific Council of body donation center of Grenoble. In this perspective, a thermostatic water tank was designed to conduct ex vivo planar tension tests in a physiological environment, i.e., in a saline solution at a temperature of $37 \pm 1{ }^{\circ} \mathrm{C}$. In order to observe the anisotropy of the tissues, the samples were tested in two directions. Tests consisting of a series of load-unload cycles of increasing amplitudes were performed to highlight their viscous behavior. The results were then discussed according to the microstructure of tissue, which was investigated using different staining methods and histological analysis. The observed behaviors were then fitted using an anisotropic hyperelastic or a visco-hyperelastic matrix-fiber model.
\end{abstract}

Keywords Urethra $\cdot$ Mechanical behavior $\cdot$ Microstructure $\cdot$ Anisotropy $\cdot$ Material modeling

\section{Introduction}

The human body is composed of different types of tissues. In anatomy, soft tissues connect, support or surround other structures and organs of the body. They can be connective tissues, such as tendons, ligaments, fascia, skin, fibrous tissues, fat, and synovial membranes, or, non-connective tissues like muscles, nerves and blood vessels (Abé and Hayashi 1996). In the field of engineering, the understanding of the mechanical macroscopic behavior of such tissues is primordial in order to design and develop novel treatment solutions.

Nowadays, different in vivo characterization methods exist. Some have the benefit of being noninvasive, such as elastography which estimates the elasticity of the tissue

\section{G. Chagnon}

gregory.chagnon@univ-grenoble-alpes.fr

1 University Grenoble Alpes, CNRS, CHU Grenoble Alpes, Grenoble INP, TIMC-IMAG, F-38000 Grenoble, France

2 Institut de Biologie et de Pathologie, Centre Hospitalier Grenoble-Alpes, 38000 Grenoble, France

3 Laboratoire d'Anatomie des Alpes Françaises, Université Grenoble-Alpes, 38000 Grenoble, France

4 Département de chirurgie digestive et de l'urgence, Centre Hospitalier Grenoble-Alpes, 38000 Grenoble, France
(Ophir et al. 1991). Other techniques, like nano-indentation (Samur et al. 2007) or aspiration devices (Nava et al. 2004), are a bit more invasive. These methods, however, only allow for simple tests, unlike the classical ex vivo characterization which allows the observation of more complex behaviors, like large strain deformation or viscoelasticity.

The mechanical behavior of most tissues and organs has already been extensively studied (Abé and Hayashi 1996), but the urinary tract remains an exception. There is a close relationship between the mechanical performance of the urethra and its physiological function during micturition. Also, most pathologies, such as hyperplasia or urinary incontinence (Chung et al. 2012), are treated using medical devices that interact with the urethra. Therefore, knowledge of its mechanical behavior can improve the design of such devices. To the authors' knowledge, a single study provides a simplified linear elastic modeling of the human urethra (Müller et al. 2008). All the other available data were produced on animals. The similarities between the sheep, the human and the porcine urethra were studied (Marti et al. 2006; Müller et al. 2010), and it was concluded that the pig's urethra best resembles the human one. A mechanical characterization of sow tissues was also proposed (Müller et al. 2008), but it seems to be anatomically different from humans (Dass et al. 2001). Natali et al. (2016) studied the mechanical behavior 
of horse urethra. To this day, this study provides the most relevant mechanical data. Other authors investigated smaller animals, such as rats, rabbits or dogs (Joller et al. 2012; Lalla and Danielsen 2007; Haworth et al. 2011; Feng et al. 2010; Lecamwasam et al. 1999).

In terms of histological and morphological description, most of the urinary tract (Tonar et al. 2004) has already been investigated, i.e., the ureters (Velardo 1981), the bladder (Dixon and Gosling 1983) and the kidneys (Rouiller 1969). The data concerning the urethra however, in comparison with the previously mentioned organs, are lacking.

This work aims, on the one hand, at observing the microstructure of the urethra of male humans by means of histological analysis. On the other hand, the mechanical response of the tissue is evaluated using experimental tensile tests. The results of the mechanical tests are discussed and explained using the histological observations, and different mechanical models (Gasser et al. 2006) accounting for hyperelastic or cyclic material behaviors are then tested in order to fit the experimental data.

\section{Experimental methods}

In this section, the anatomy of the urethra is described and the sample extraction procedure is explained. The histological methods used to analyze the microstructure of tissue are then detailed. Finally, the experimental testing apparatus is presented.

\subsection{Anatomical description of male urethral soft tissue and sample extraction}

The male urethra, as presented in Fig. 1b, is $18-20 \mathrm{~cm}$ long and extends from the internal orifice in the urinary bladder to the external opening, or meatus, at the end of the penis. It consists of four continuous parts, which are numbered 1-4 in Fig. 1b. The pre-prostatic urethra (part 1) is approximately $1 \mathrm{~cm}$ in length and extends from the base of the bladder to the prostate. The prostatic (part 2) urethra is $3-4 \mathrm{~cm}$ in length and tunnels through the substance of the prostate. The membranous (part 3) part of the urethra is about $2 \mathrm{~cm}$ in length and the least dilatable part. It descends with a slight ventral concavity from the prostate to the bulb of the penis, passing through the perineal membrane.

The anterior part or spongy urethra (part 4) lies within the corpus spongiosum penis. In the flaccid penis, it is about $15 \mathrm{~cm}$ long and extends from the end of the membranous urethra to the external urethral orifice on the glans penis. It starts below the perineal membrane at a point anterior to the lowest level of the symphysis pubis as the bulbar urethra (Ellis 2005; Standring 2015).

\subsection{Sample extraction and methodology}

Formalin-fixed cadavers were selected for anatomical dissection from the Anatomy laboratory, faculty of medicine, Grenoble, France (Laboratoire d'Anatomie Des Alpes Françaises). Cadavers were embalmed with a formalin solution (ARTHYL) injected into the carotid artery and drained from the jugular vein, and then preserved in a refrigerated room. Cadavers were placed on their backs; legs spread in the frog position. The scrotum was pulled upward to stretch the skin of the perineum. A horseshoe perineal incision was performed and carried down to the urethra, which was then dissected free from surrounding tissues. The external portion of the urethra (outside the human body) and the bulbospongiosus muscle were initially taken using a distal section upstream of the fossa navicularis. The interior part (inside the human body) was then dissected along its posterior face to the apex of the prostate, where the proximal section was performed. The remaining tissue was then cut along its longitudinal direction as illustrated in Fig. 2a. Figure 1a shows the tissue prepared before specimen extraction. Our study includes 8 anatomical dissections performed on human male cadavers, without apparent urethral pathology or scars, aged between 72 and 87 years (median age 84 years), with heights between 160 and $180 \mathrm{~cm}$ (mean height $170 \mathrm{~cm}$ ), and weighing between 70 and $80 \mathrm{~kg}$ (mean weight $77 \mathrm{~kg}$ ). Five of the extracted samples were used for mechanical testing, and three were included in paraffin for histological observations. All the tests were performed in compliance with French regulations on postmortem testing, and the protocol was approved by a local scientific committee of the Grenoble Alpes University. Evidently, the number of samples in the present study is limited for statistical analysis, but sufficient to ensure reproducibility. In both histological analysis and mechanical testing, very little dispersion was observed. For these reasons, values obtained on the most relevant samples will be presented instead of average and standard deviation values.

\subsection{Histology}

Three urethrae with no apparent anomalies were chosen for histological analysis. Samples were conserved in formaldehyde, fixed first in formalin $10 \%$ during $24 \mathrm{~h}$ at $4{ }^{\circ} \mathrm{C}$ and then embedded in paraffin. The samples used for histological observations are shown on the simplified illustration of the urethral duct presented in Fig. 1b: cuts in the transverse ( $\mathrm{A}, \mathrm{B}$ and $\mathrm{C}$ for the membranous urethra, $\mathrm{G}, \mathrm{F}$ and $\mathrm{H}$ for the spongy urethra) and coronal (D and $\mathrm{E}$ for the membranous urethra, I and $\mathrm{J}$ for the spongy urethra) planes. Sections of $3 \mu \mathrm{m}$ were then cut. The slides were then stained with Hematoxylin Eosin Saffron (HES) to see 


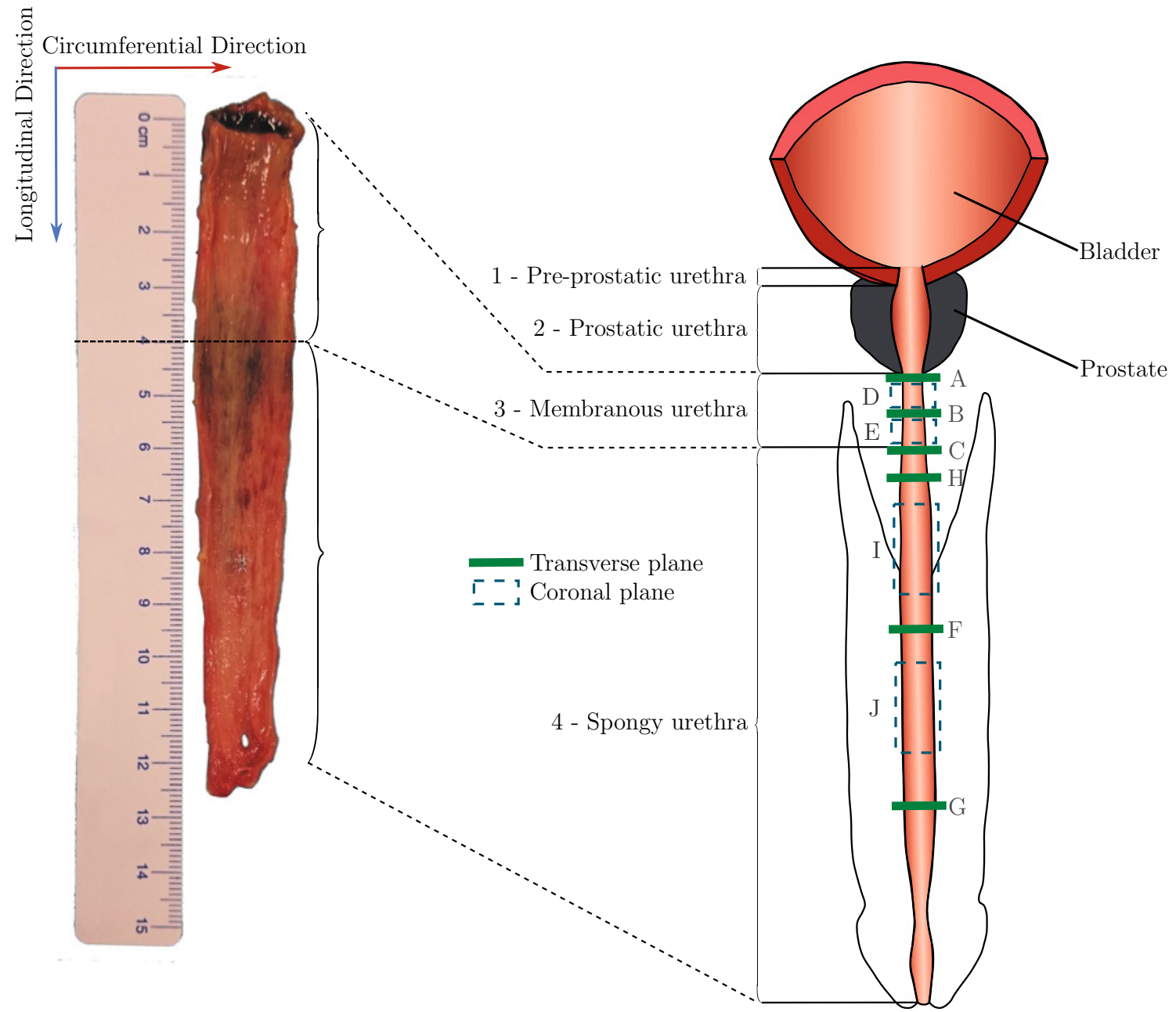

(a)

(b)

Fig. 1 a Urethra after longitudinal incision, b illustration of the corresponding portions of the urethra on an anatomical diagram. The different planes of cuts used for histological measures are also shown

nucleic acids and connective tissue, Verhoeff's staining to highlight elastin fibers and sirius red to observe all types of collagen and muscular fibers. An immunohistochemistry study was also performed. Staining analysis was also conducted in order to highlight the different types of collagen fibers. Sections were dewaxed, rehydrated, and the antigen retrieval was performed with a preheated protease. They were stained using a type I human anti-collagen diluted to the twentieth in rabbit polyclonal for $1 \mathrm{~h}$ at $37^{\circ} \mathrm{C}$. Antibody binding was detected using the ultraView Universal DAB Detection kit (Automate Benchmark XT IHC/ISH (ROCHE-VENTANA) 760-500) and 3,3-diaminobenzidine (DAB) as chromogen. Slides were counterstained with hematoxylin. Pictures were acquired by a digital camera (Leica Microsystems) connected to an optical microscope (Leica Microsystems) and processed with the Leica Application Suite software tools. Orientations of muscle, elastin and collagen were inspected.

\subsection{The mechanical tests}

The samples were tested within the hour of explantation, during which the samples were preserved in a saline solution. The tests were carried out by means of a GABO Eplexor $500 \mathrm{~N}$ machine fitted with a thermo-regulated tank filled with $0.9 \%$ sodium chloride solution and a $10 \mathrm{~N}$ force transducer (Breche et al. 2016). The saline solution was heated to a temperature of $37 \pm 1{ }^{\circ} \mathrm{C}$.

The urethra was cut and unraveled along its longitudinal direction, as illustrated in Fig. 2a. Rectangular specimens of $34.56 \pm 5.05 \mathrm{~mm}$ width, $2.53 \pm 0.24 \mathrm{~mm}$ thickness and 10 $\mathrm{mm}$ height were gathered from the membranous and spongy portions of the urethra along the longitudinal and circumferential directions as shown in Fig. $2 b$.

The samples were mounted in a rigid structure containing clamps outside the tank in order to ensure a consistent geometry for the samples, i.e., a fixed height of $2 \mathrm{~mm}$ and 


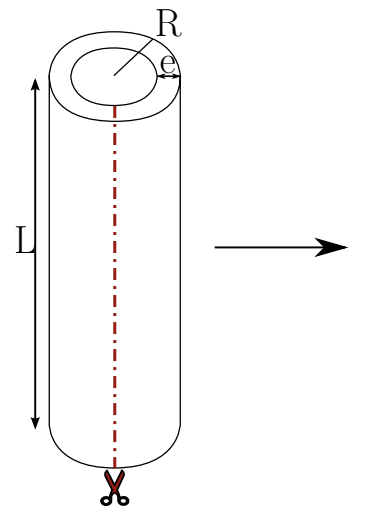

(a)

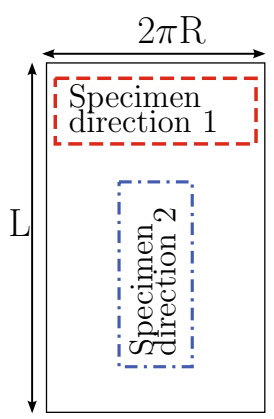

(b)

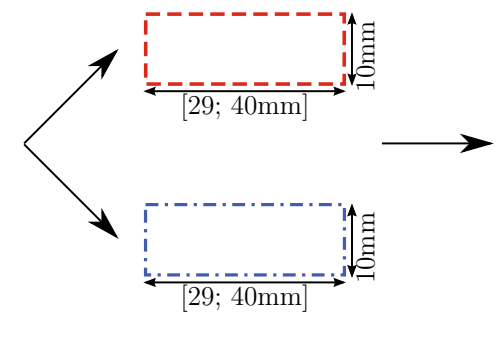

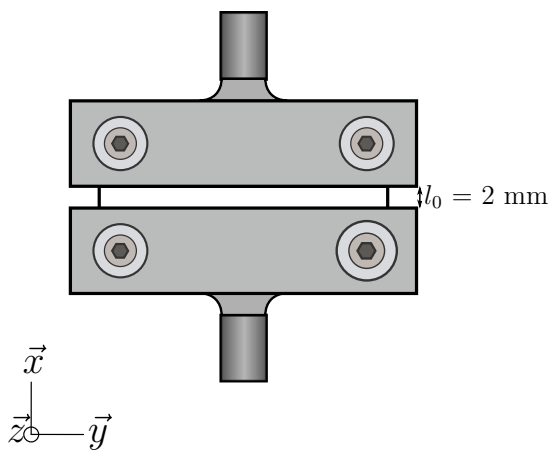

(c)
Fig. 2 a The urethra cut along its longitudinal direction. b Planar tension specimens are gathered from the unfolded urethra. Specimen direction 1 is used to evaluate the behavior of the tissue in the longitu- dinal direction. Specimen direction 2 is used to evaluate the behavior of the tissue in the circumferential direction. $\mathbf{c}$ The specimen is inserted in the clamps before transfer into the thermostatic tank

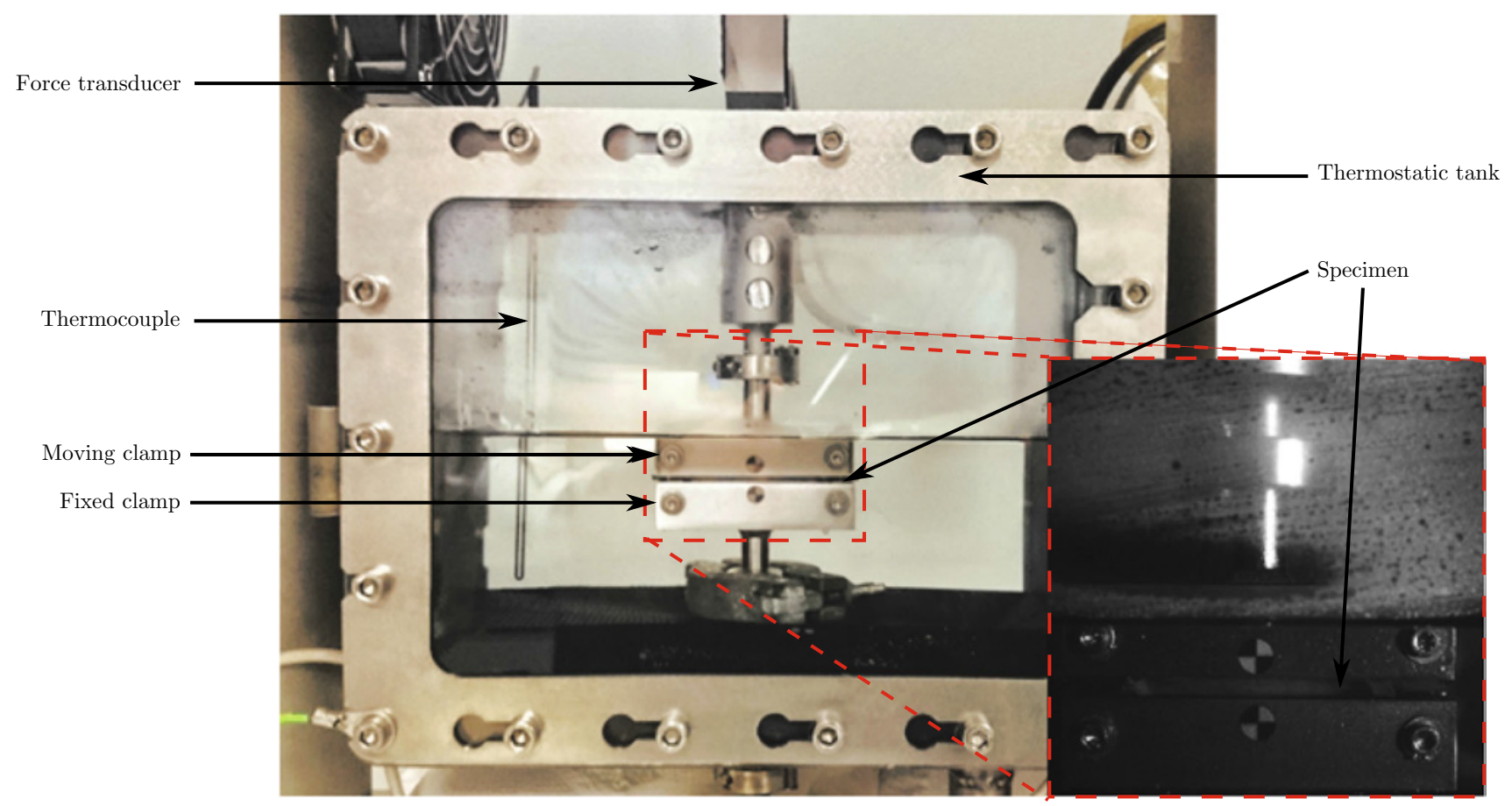

Fig. 3 Thermostatic tank fitted on a GABO Eplexor $500 \mathrm{~N}$ to allow immersed testing

a width large enough $(\geq 30 \mathrm{~mm})$ so that the samples stay rectangular throughout the tests. Figure $2 \mathrm{c}$ shows the rigid clamps-sample assembly which was then transferred into the testing apparatus. Figure 3 shows the final configuration of the machine before running the test.

The membranous and the spongy portions of the urethra were tested in both the longitudinal and circumferential directions. During the test, it is supposed that the sample stays rectangular and border effects are neglected (Charlton et al. 1994). The deformation gradient tensor $\overline{\bar{F}}$ describes the motion of a continuum. It characterizes the transformation of a material point from the reference configuration to a deformed one. Non-porous soft tissues are classically considered incompressible (Holzapfel 2000), which translates into $\operatorname{det}(\overline{\bar{F}})=1$. This implies that in planar tension condition $\overline{\bar{F}}$ can be written as:

$[\overline{\bar{F}}]=\left[\begin{array}{ccc}\lambda & 0 & 0 \\ 0 & 1 & 0 \\ 0 & 0 & \lambda^{-1}\end{array}\right]$ 

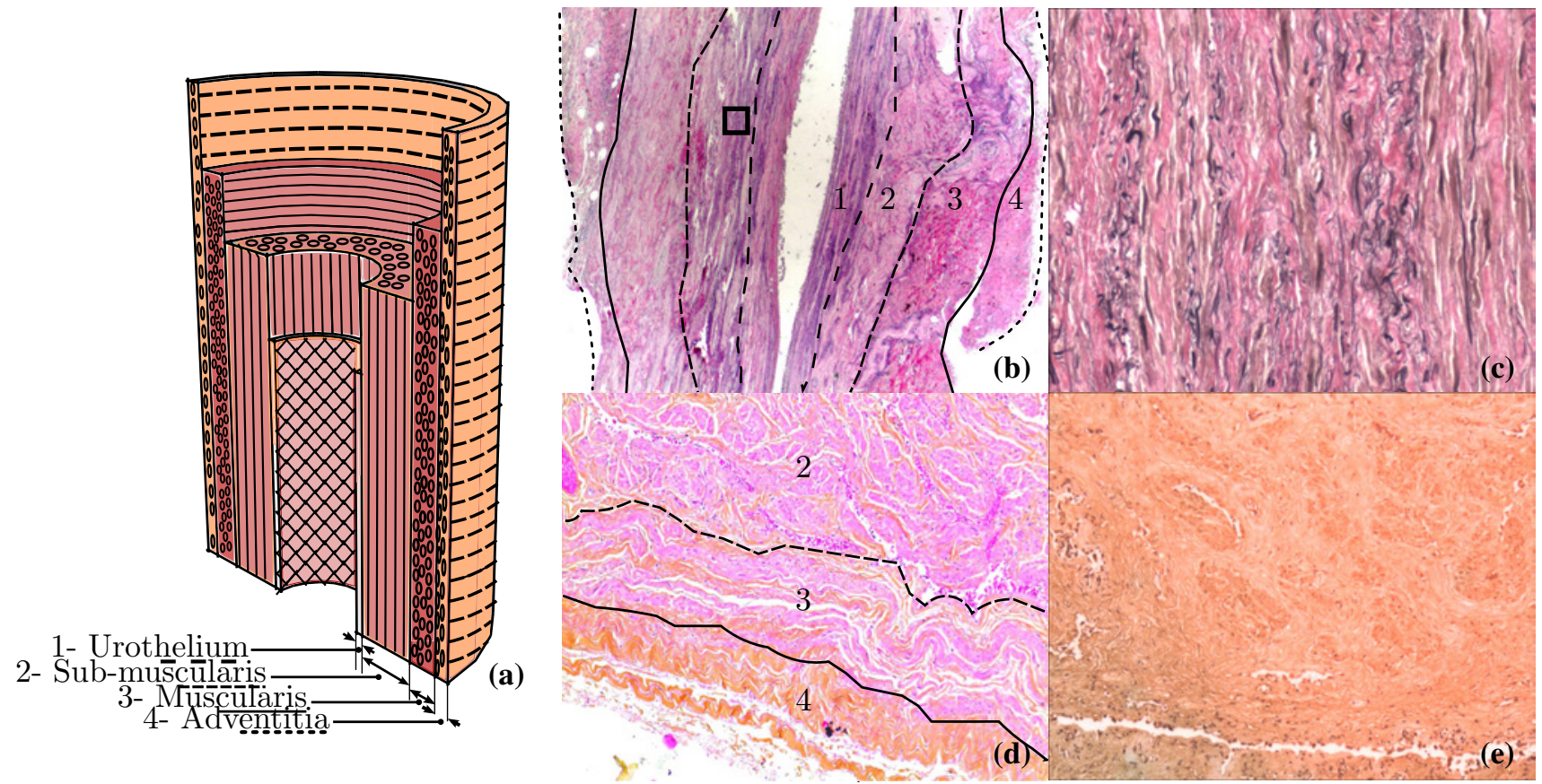

Fig. 4 a 3D illustration of the urethra of a male subject. Only the 4 main layers are represented along with the main fiber directions. b Histological cut in the coronal plane using Verhoeff's staining agent (zoom $\times 1.6$ ). The square delimits the viewing field of figure $\mathbf{c}$. $\mathbf{c}$ Histological cut in the coronal plane using Verhoeff's staining agent $($ zoom $\times 20)$. d Hematoxylin Eosin Saffron staining in the transverse plane (zoom $\times 5$ ). e Immunohistochemical staining in the transverse plane (zoom $\times 5$ ). The horizontal split at the bottom of the image corresponds to the lumen of the urethra where $\lambda=\frac{l}{l_{0}}$ is the stretch $\left(l\right.$ and $l_{0}$ are the current and initial height of the specimen, as can be seen in Fig. 2c). Each sample underwent the same cyclic planar tension test consisting of a series of loading and unloading phases of increasing stretch levels, namely 1.1, 1.2, 1.3, 1.4, 1.5, 1.75, 2.0 at a constant strain rate of $5.10^{-3} \mathrm{~s}^{-1}$. The load case is shown in Fig. 6. The maximal 2.0 stretch level was chosen since it was the highest amplitude for which most specimens remained undamaged. The tests are analyzed by measuring the nominal stress defined as:

$\Pi=\frac{F}{A_{0}}$

where $F$ is the force and $A_{0}$ the initial cross section.

\section{Results}

\subsection{Histological analysis of the male human urethra}

Starting from the lumen to the outer diameter of the tissue in the transverse plane, the spongy urethra is composed of four layers: a submucosa, shown in Fig. 4(a, b-urothelium) consisting of a urothelium and a $50 \mu \mathrm{m}$ conjunctive tissue containing capillaries. A spongy tissue composed of void vascular spaces close to endothelial cells associated with longitudinal smooth muscle fibers. These fibers are bundled into masses associated with many collagen and elastic fibers disposed in a circular motion, which form a $100 \mu \mathrm{m}$ layer that can be seen in Fig. 4(a, b, d-submuscularis). Figure $4 \mathrm{c}$ shows the direction of the elastic fibers in this portion of the tissue. A third layer-the external muscularis-measuring approximately $80 \mu \mathrm{m}$ consists of thin, discontinuous muscular cells of circular disposition. It is associated with an abundant extracellular matrix composed of circular collagen fibers which are shown in Fig. 4(a, b, d-muscularis). Everything is surrounded by an adventitia composed of loose conjunctive tissue containing vascular and nervous axes [cf. Fig. 4(a, b, d-adventitia)]. The immunohistochemical analysis presented in Fig. 4e shows that the tissue in general is rich in type I collagen fibers.

Geometrically, although the angle of orientation of the muscle fibers of the different layers in relation to the axis of the lumen is difficult to measure, it appears that the transverse and longitudinal muscle fibers are orthogonal: in Fig. 4(b-layer 2), the muscle fibers are bundled together (pink coloration) and surrounded by collagen fibers (orange coloration). The bundles are discontinuous, and the nuclei of the muscular cells appear as dots which suppose a trans- 


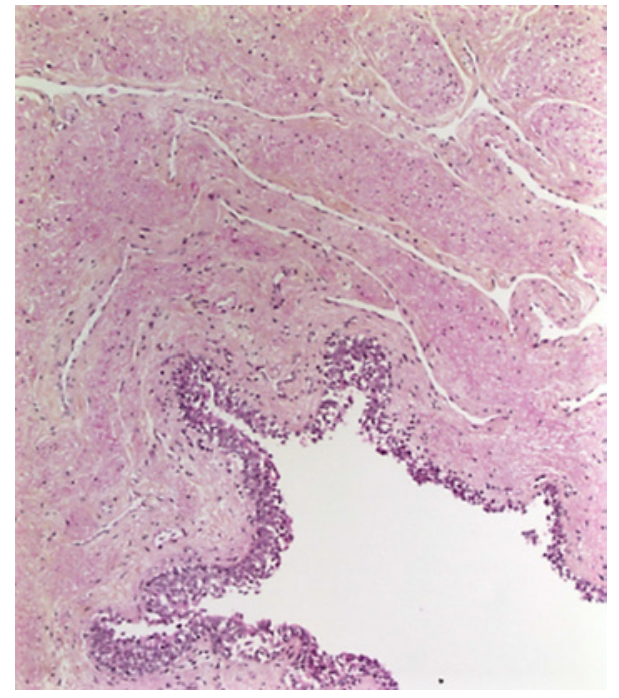

Fig. 5 Observation of a portion of the lumen of the membranous urethra

verse incision. In the transverse muscle layer, however, the cells are continuous and their nuclei appear to be oval. This is due to an incision in their longitudinal direction which implies that this layer is composed of circular muscle fibers. Figure $4 \mathrm{~d}$ gives a representation of the urethral tissue of male subjects. All the observations made on the spongy urethra apply to the membranous urethra, the only difference being a thicker muscularis mucosa, which seems logical considering the near presence of the striated sphincter.

Figure 5 shows the lumen of the membranous urethra, the urothelium and the sub-muscularis layer of the urethra. The random disposition of the folds of the urothelium is similar to those of the equine urethrae observed in the literature (Natali et al. 2017a,b).

\subsection{Mechanical behavior of the human male urethra}

The histological observations prove that the specimens were cut in the symmetry axes of the materials, ensuring that the planar tensile test hypothesis is respected. Figure 6 shows the results for both planar tension test directions of the membranous urethra. The behavior is quite similar for both directions. Only one representative result is presented in each figure as the results on the different anatomical dissections were very close. The largest standard deviation observed evaluated to $15 \%$ and was on average equal to $8.9 \%$. First by observing the first loads only, the tissues present a nonlinear response to an applied strain. Second, a hysteresis is observed, which is characteristic of the viscoelastic behavior of the urethra. Third, considering two successive loading cycles, a loadunload cycle to 1.1 followed by one reaching 1.2 for example, the hysteresis mentioned earlier is observed, but the curve of the second load is different than the first load. Instead, a smaller stress is generated until reaching the strain limit of the first cycle, after which the curve seems to follow the path of the loading phase of the first cycle. This phenomenon is observed throughout all the cycles. In general, the hysteresis increases with the applied load, which in turn generates increased residual strain. Residual strain is characterized by stress reaching zero while strain is still positive. It increases with the maximum strain applied to the material throughout the cycles and is larger in the longitudinal direction. No conclusions can be deduced on the possibility to recover this residual strain due to the limited number of specimens. It is important to note that the value of the stress lower than 0 should not be trusted as the specimen is compressed and local buckling occurs. The difference between maximum stress for both directions at the end of the load cycles gives an idea about anisotropy. Although the difference varies very slightly

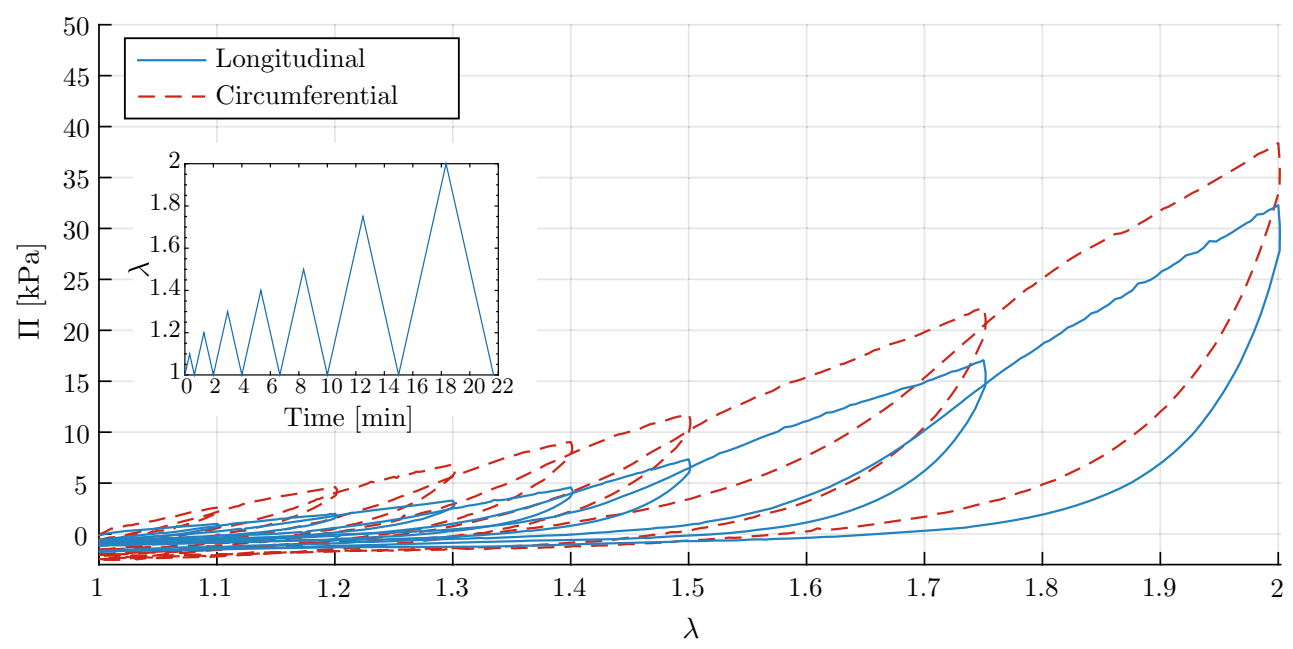

Fig. 6 Experimental results of planar tension tests in both directions of the membranous urethra 


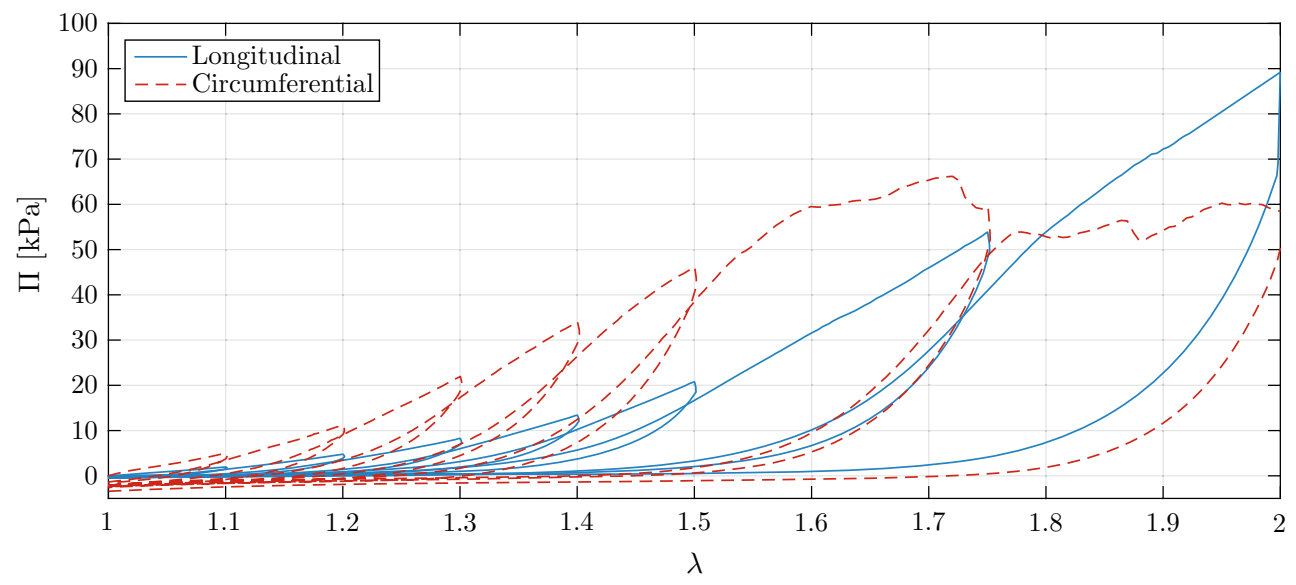

Fig. 7 Experimental results of planar tension tests in both directions of the spongy urethra

(from 2 to $10 \mathrm{kPa}$ ), the ratio decreases from 2 at a 1.1 stretch to 1.15 for a stretch of 2 .

The results observed for the spongy urethra in Fig. 7 present less similarities in the two loading directions. The samples are stiffer in the circumferential direction although some local failures were observed after a stretch of 1.6. The phenomena observed for the membranous part of the urethra, i.e., hysteresis, residual strain and anisotropy, also apply in this case: residual strain is larger in the circumferential direction, as opposed to the membranous portion of the urethra, but hysteresis and anisotropy are more important. The maximum stress of each loading cycle is also at least twice larger than the one observed earlier.

Müller et al. (2008) report Young moduli of 5, 11 and 21 $\mathrm{kPa}$ at, respectively, 15, 35 and $45 \%$ strains, which corresponds to stresses of $0.75,3.85$ and $9.45 \mathrm{kPa}$. These values are close to those observed on the membranous urethra in the longitudinal direction. However, the comparison is difficult since the tested portion of the urethra, the preservation method of the cadaver and the direction of testing are not specified in their study.

In comparison with data observed on equine urethrae (Natali et al. 2017a, b), the human urethra is stiffer. Also the urethra seems stiffer in the circumferential direction, which is the opposite of what can be observed on the equine tissue. The anisotropy, however, is close in both directions for both subjects.

\section{Discussion}

The general behavior of the male urethra in both directions can be explained by its physiological functions. On the first hand, the urethra is an evacuation duct that plays a key role in the micturition process, which can lead to intra-urethral pressures reaching $200 \mathrm{~cm} \mathrm{H}_{2} \mathrm{O}$ (Giannantoni et al. 2003). It thus seems natural that the urethra is more resistant in the circumferential direction. On the other hand, it is strongly loaded by the dilatation of the corpus cavernosum during erection (Bochereau et al. 1996), during which the urethra endures large deformations in the longitudinal direction. This explains its softer behavior in this direction. The histological observations also concur with the previous statements. The adventitia and the muscularis mucosa are essentially composed of circular collagen and smooth muscle fibers. Combined, their thickness is larger than the one of the submuscularis, thus providing more resistance to tension. Also, Fig. 4 shows that the elastic fibers are mostly aligned in the longitudinal direction in the sub-muscularis and in the circumferential direction in the muscularis. By considering an average lumen size of $40 \mu \mathrm{m}$ and a $1 \mathrm{~cm}$ portion in length, the volume of each can be calculated. Although the former $(100 \mu \mathrm{m})$ is thicker than the latter $(80 \mu \mathrm{m})$, the volume of the sub-muscularis evaluates to $87.9 \mathrm{~mm}^{3}$ while the muscularis evaluates to $115.6 \mathrm{~mm}^{3}$, thus explaining the stiffer behavior in the circumferential direction. In this study, it is supposed that the muscularis and the sub-muscularis contribute the most to the mechanical behavior of the tissue. The knowledge of the orientation of their principal components allows for the modeling of the anisotropic behavior of the material.

\subsection{Hyperelastic modeling}

In this section, it is proposed to fit the experimental data using a hyperelastic material model already established in the literature in order to reproduce the equivalent mechanical response of the urethra. As a first approach, the viscous behavior and thus the hysteresis will not be taken into account. Also, the consecutive first loads only are evaluated. In a second approach, the cyclic behavior will be studied and an adapted constitutive equation is fitted. The Cauchy stress 


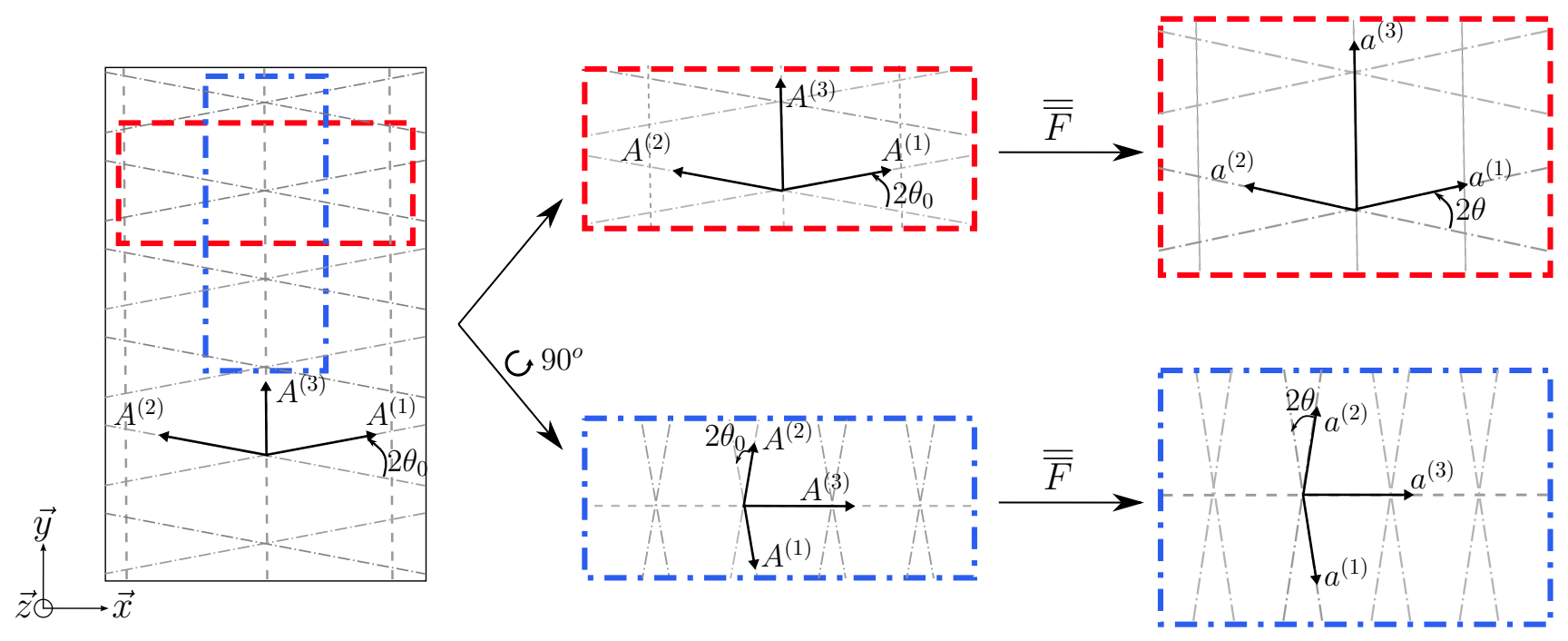

Fig. 8 Drawing illustrating the principle of anisotropy

tensor with the incompressibility hypothesis can be written as follows:

$\overline{\bar{\sigma}}=-p \overline{\bar{I}}+2 \overline{\bar{B}} \frac{\partial W}{\partial \overline{\bar{B}}}$

where $\overline{\bar{I}}$ represents the identity tensor, $p$ the hydrostatic pressure, $W$ the strain energy function, and $\overline{\bar{B}}$ the left Cauchy-Green strain tensor which evaluates to $\overline{\bar{F}} \overline{\bar{F}}^{T}$.

The strain energy function of the histological based anisotropic model established by Gasser et al. (2006) was chosen to describe the anisotropic behavior of the material.

$W=\mu\left(I_{1}-3\right)+\frac{k_{1}}{2 k_{2}} \sum_{\alpha}\left(e^{k_{2}\left[\kappa\left(I_{1}-3\right)+(1-3 \kappa)\left(I_{4}^{\alpha}-1\right)-1\right]^{2}}-1\right)$

where $\mu$ and $k_{1}>0$ are stress-like parameters, $k_{2}>0$ is a dimensionless parameter, and $\kappa$ takes into account the dispersion of fiber orientation. It is worth noting that $\kappa$ can only take values in the $\left[0, \frac{1}{3}\right]$ range, $\frac{1}{3}$ corresponding to an isotropic fiber distribution. The strain invariants $I_{1}$ and $I_{4}$ can be written as:

$$
\begin{aligned}
& I_{1}=\operatorname{tr}(\overline{\bar{B}}) \\
& I_{4}{ }^{(\alpha)}=\overline{\bar{A}}^{(\alpha)} \overline{\bar{F}}^{T} \overline{\bar{F}} \bar{A}^{(\alpha)}
\end{aligned}
$$

where $\bar{A}^{(\alpha)}$ is the initial direction of each set of fibers in a fiber reinforced material. In the deformed state, the orientation at any given time can be written as $\bar{a}^{(i)}=\overline{\bar{F}} \bar{A}^{(i)}$. Figure 8 shows the sample in its reference and deformed states. The fiber orientation was chosen according to the histomorphometric conformation observed. Three directions were chosen. Directions (1) and (2) correspond to the fibers in the muscularis (refer to Layer (3) of Fig. 4) and direction (3) corresponds to the fibers in the sub-muscularis (refer to Layer (2) of Fig. 4). The fibers of the other layers are supposed not to influence the mechanical behavior of the tissue. It is assumed in this study that the fibers only react in tension. The orientation angle $\theta$ (Fig. 8) is, respectively, estimated to $5^{\circ}$ and $2^{\circ}$ for the membranous and spongy parts of the urethra.

\subsubsection{Modeling the response of the tissue}

For the membranous portion of the urethra (Fig. 9), the anisotropy decreases while the nonlinearity increases. The data are well fitted, especially over a stretch of 1.5. Under this threshold, the fit is slightly less accurate.

In the case of the spongy urethra (Fig. 10), although some of the experimental data are compromised due to local failures during the test for the circumferential direction, both the nonlinearity and the anisotropy ratios increase. This part of the tissue is also a lot stiffer than the membranous part. The constitutive model does a great job fitting the data.

Table 1 provides the values of the material parameters of the constitutive model for both portions of the tissue. The values of the parameters ensure that the incompressibility hypothesis does not alter the model simulations (Toungara et al. 2012).

\subsection{Cyclic modeling}

As a second approach, the viscoelastic behavior and the stress softening of the material are taken into account. In this modeling, the fiber directions are considered oriented at 0 and $90^{\circ}$. It is also assumed that the material is composed of four parts: 


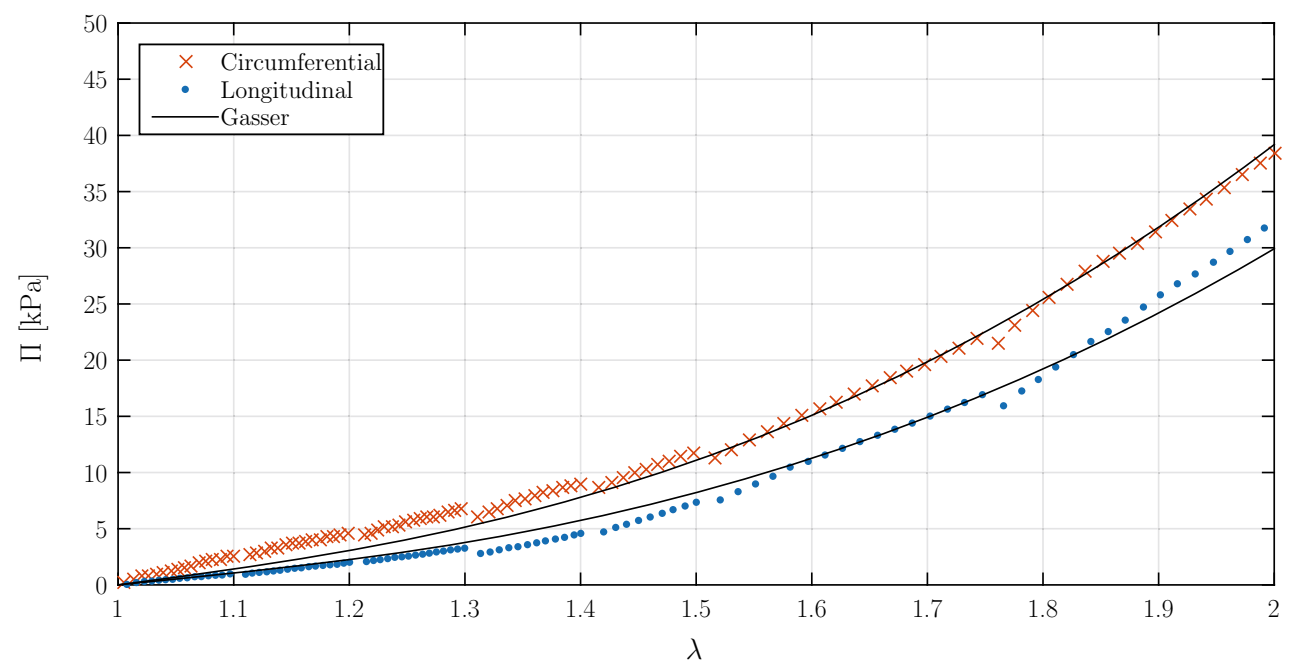

Fig. 9 Experimental data of the membranous urethra fitted using the Gasser et al. anisotropic model

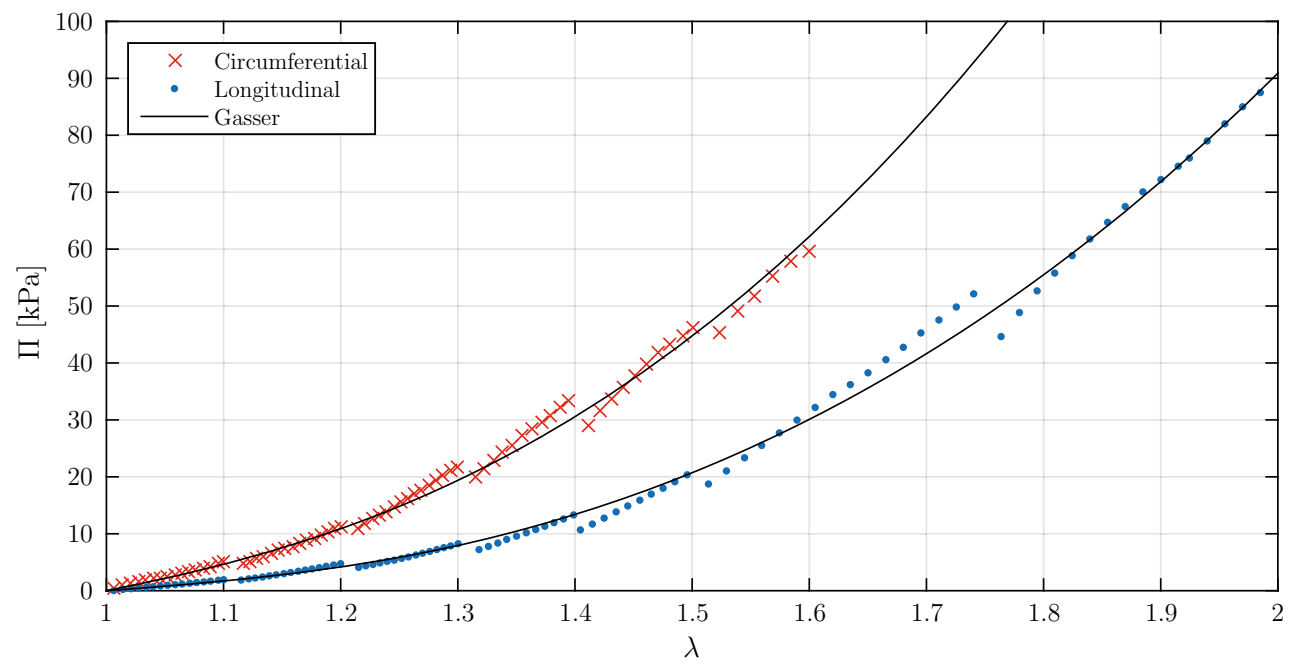

Fig. 10 Experimental data of the spongy urethra fitted using the Gasser et al. anisotropic model

Table 1 Material parameter values for both portions of the urethra for a large strain load

\begin{tabular}{lll}
\hline & Membranous urethra & Spongy urethra \\
\hline Gasser et al. (2006) & $\mu=3.65 ; k_{1}=1.88 \mathrm{kPa} ;$ & $\mu=5.79 ; k_{1}=7.77 \mathrm{kPa} ;$ \\
& $k_{2}=0.001 ; \kappa=0.206 ; \alpha=3$ & $k_{2}=0.001 ; \kappa=0.3 ; \alpha=3$ \\
\hline
\end{tabular}

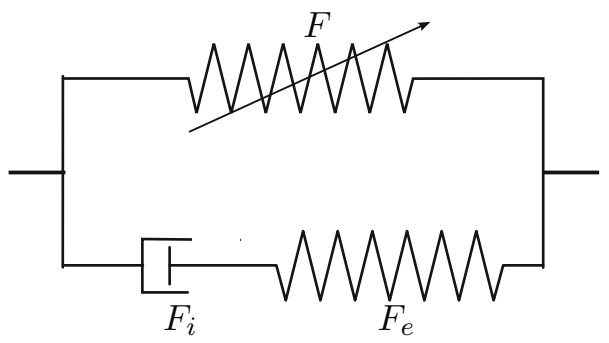

the three different fiber layers and the soft tissue matrix. It can be considered that the mechanical response of the material will be the addition of each contribution. As in the previous part, the matrix is considered as perfectly elastic. The fibers are considered to behave like unidimensional element with no bending stiffness, meaning that they are only subject to uniaxial tension whatever the loading is. The matrix is modeled by a Neo-Hookean material, and regarding the experimental results, it is now considered that the fibers present a viscoelastic mechanical behavior with stress softening. Their mechanical behavior can be represented by the rheological scheme presented in Fig. 11.
Fig. 11 Rheological representation of the viscoelastic model

It is a Zener model, where the elastic part is replaced by an elastic model with stress softening. The inelastic branch 
Table 2 Parameter values of the viscoelastic anisotropic model. $C_{1}, k_{1}, C_{10}, C_{20}, C_{30}$ are expressed in $k P a . \eta_{0}$ and $\eta_{m}$ are expressed in $\mathrm{Pa}$ s.

\begin{tabular}{|c|c|c|c|c|c|c|c|c|c|c|}
\hline & \multirow[t]{2}{*}{ Direction } & \multirow[t]{2}{*}{$C_{1}$} & \multicolumn{4}{|l|}{$F$} & \multirow{2}{*}{$\begin{array}{l}F_{i} \\
\eta_{0}\end{array}$} & \multicolumn{3}{|c|}{$F_{\mathrm{e}}$} \\
\hline & & & $k_{1}$ & $k_{2}$ & $\eta_{m}$ & $\beta$ & & $C_{10}$ & $C_{20}$ & $C_{30}$ \\
\hline \multirow[t]{3}{*}{ Membranous urethra } & Matrix & 0.1 & - & - & - & - & - & - & - & - \\
\hline & $A^{(1)}, A^{(2)}$ & - & 2.75 & 0.001 & 2 & 0.6 & 0.15 & 10 & 0.1 & 0.001 \\
\hline & $A^{(3)}$ & - & 2.5 & 0.1 & 2 & 0.6 & 0.08 & 10 & 0.1 & 0.001 \\
\hline \multirow[t]{3}{*}{ Spongy urethra } & Matrix & 0.1 & - & - & - & - & - & - & - & - \\
\hline & $A^{(1)}, A^{(2)}$ & - & 0.75 & 0.2 & 1.5 & 0.6 & 0.15 & 10 & 0.1 & 0.001 \\
\hline & $A^{(3)}$ & - & 5.8 & 0.2 & 1.8 & 0.75 & 0.08 & 10 & 0.1 & 0.001 \\
\hline
\end{tabular}

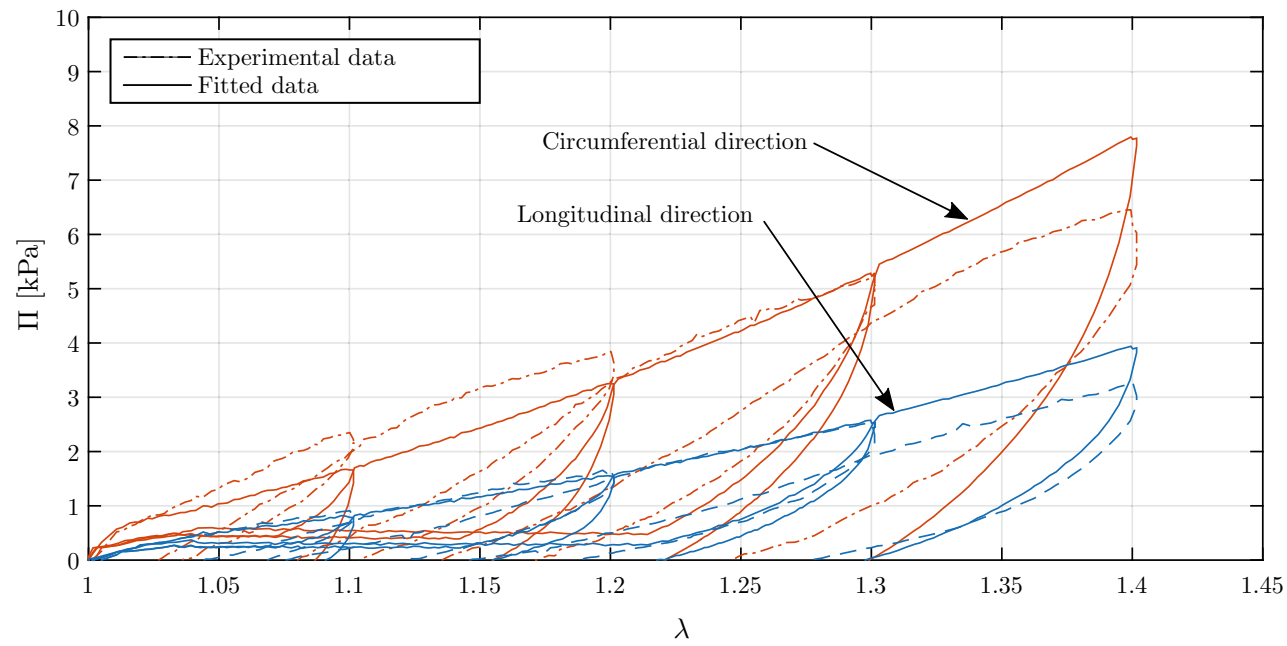

Fig. 12 Experimental data of the membranous urethra fitted using the viscoelastic anisotropic model

is described by the de decomposition of the deformation gradient into an elastic part $\overline{\overline{F_{\mathrm{e}}}}$ and an inelastic one $\overline{\overline{F_{i}}}$ with $\overline{\bar{F}}=\overline{\overline{F_{\mathrm{e}}}} \overline{\overline{F_{i}}}$. Different constitutive equations can be used to describe the different parts of the model. For the elastic part, a classical Holzapfel et al. (2000) model is used:

$W_{0}=\frac{k_{1}}{k_{2}}\left(\exp k_{2}\left(I_{4}-1\right)^{2}-1\right)$

$k_{1}$ and $k_{2}$ are material parameters. A stress softening function must be introduced to capture the difference between the two first loads. Different approaches can be used as pseudoelasticity (Peña and Doblare 2009) as internal variables (Ehret and Itskov 2009), damage (Maher et al. 2012) or double network theory (Rebouah and Chagnon 2014a). This last method is used. It is based on Rebouah et al. (2013) model: a multiplicative function that depends on the maximum value reached by $I_{4}$, noted $I_{4}^{\max }$, is added to the strain energy density that becomes

$W=F\left(I_{4}^{(\alpha)}, I_{4}^{(\alpha) \max }\right) W_{0}$

The functions is expressed as:

$F\left(I_{4}^{(\alpha)}, I_{4}^{(\alpha) \max }\right)=1-\eta_{m}\left(\frac{I_{4}^{(\alpha) \max }-I_{4}^{(\alpha)}}{I_{4}^{(\alpha) \max }-1}\right)^{\beta}$ if $I_{4}^{(\alpha) \max } \neq 1$, in the other cases the function evaluates to 1 . In the other branch, the spring is modeled by a Yeoh (1993) constitutive equation and the dashpot is simply linear with a damping parameter called $\eta_{0}$. In uniaxial extension, the general form of the evolution of $\overline{\overline{F_{\mathrm{e}}}}$ was given by Petiteau et al. (2013) and Rebouah and Chagnon (2014b) and is:

$\dot{\lambda}_{\mathrm{e}}=\lambda_{\mathrm{e}} \frac{\dot{\lambda}}{\lambda}-\frac{4}{3 \eta_{0}} \frac{\partial W^{e}}{\partial I_{1}}\left(\lambda_{\mathrm{e}}^{3}-1\right)$

where $\lambda_{\mathrm{e}}$ represents the stretch component of $\overline{\overline{F_{\mathrm{e}}}}$, i.e., the stretch in uniaxial extension. In the case of each fiber, the previous equation is applied with $\lambda_{\mathrm{e}}=\sqrt{I_{4}^{(\alpha)}}$. The same equation is used for the three directions of fibers.

Finally, the mechanical parameters are fitted on the previous experimental data. The values of the parameters are given in Table 2.

The results of the model are presented in Figs. 12 and 13. The model describes well the hysteresis loops and the stress softening. The only difference concerns the residual strain where the model keeps a weak stress instead of 0 . In the case of the membranous urethra, as can be seen in Fig. 12, the model fits well the first 3 hysteresis loops in the longitudinal direction but slightly overestimates the last one. In the 


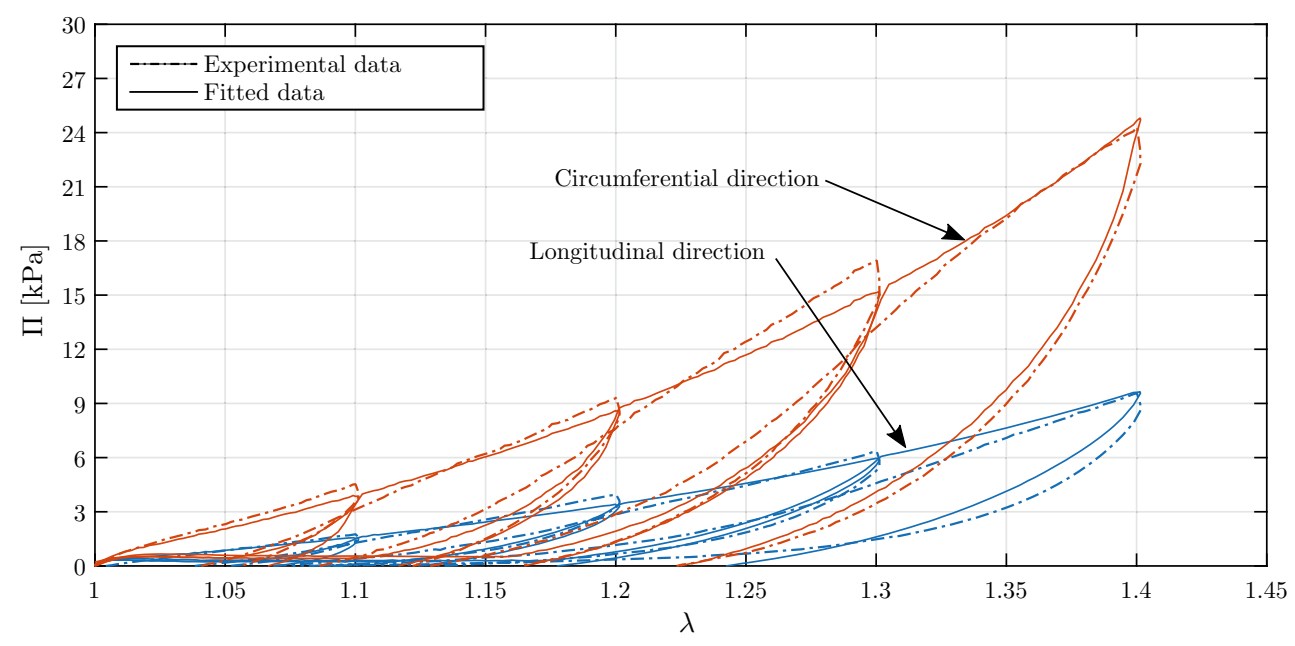

Fig. 13 Experimental data of the spongy urethra fitted using the viscoelastic anisotropic model

circumferential direction, the data are harder to fit, but the general behavior is well captured.

Concerning the spongy urethra, the model does a better job reproducing the mechanical behavior of the tissue in both directions, which is illustrated in Fig. 13.

\section{Conclusion}

This study investigated the mechanical behavior of different portions of the male human urethra. After a histomorphological analysis of the tissue, a mechanical study in a controlled environment consisting of cyclic tests was made. A tank containing a thermostatic aqueous medium was developed to best mimic the physiological condition of the tested tissues. This allowed for the observation of different phenomena such as hyperelasticity, softening and residual strain. The results show an influence of the loading direction, which is representative of an anisotropic behavior. A strong hysteresis due to the viscoelastic properties of soft tissues was also observed. As a first approach, the viscoelastic behavior was neglected and the anisotropic model established by Gasser et al. was used to fit the experimental data. These first results were promising but only applied to the first loads imposed on the material. They provide nonetheless interesting data for modeling of the human urinary tract. The second approach, which consisted in capturing the viscoelastic behavior of the material when it was subjected to cyclic deformation loads, fits the data remarkably well.

\section{Compliance with ethical standards}

Conflict of interest The authors declare that they have no conflict of interest.

\section{References}

Abé H, Hayashi K (1996) In: Sato M (ed) Data book on mechanical properties of living cells, tissues, and organs. Springer, Tokyo

Bochereau G, Cathelineau X, Buzelin JM, Bouchot O (1996) Urètre masculin, anatomie chirurgicale, voies d' abord, instrumentation. Traité Techn Chir Urol 41-305:1-26

Breche Q, Chagnon G, Machado G, Girard E, Nottelet B, Garric X, Favier D (2016) Mechanical behaviours evolution of a PLA-bPEG-b-PLA triblock copolymer during hydrolytic degradation. J Mech Behav Biomed Mater 60:288-300

Charlton DJ, Yang J, Teh KK (1994) A review of methods to characterize rubber elastic behavior for use in finite element analysis. Rubber Chem Technol 67:481-503

Chung E, Ranaweera M, Cartmill R (2012) Newer and novel artificial urinary sphincters (AUS): the development of alternatives to the current AUS device. BJU Int 112(4):5-11

Dass N, McMurray G, Greenland JE, Brading AF (2001) Morphological aspects of the female pig bladder neck and urethra: quantitative analysis using computer assisted 3-dimensional reconstructions. J Urol 165(4):1294-9

Dixon JS, Gosling JA (1983) Histology and fine structure of the muscularis mucosae of the human urinary bladder. J Anat 136(2):265

Ehret AE, Itskov M (2009) Modeling of anisotropic softening phenomena: application to soft biological tissues. Int J Plast 25:901-919

Ellis H (2005) Anatomy of the urinary bladder, prostate and male urethra. Surgery (Oxford) 23(3):97-98

Feng C, Xu YM, Fu Q, Zhu WD, Cui L, Chen J (2010) Evaluation of the biocompatibility and mechanical properties of naturally derived and synthetic scaffolds for urethral reconstruction. J Biomed Mater Res Part A 94A(1):317-325

Gasser TC, Ogden RW, Holzapfel GA (2006) Hyperelastic modelling of arterial layers with distributed collagen fibre orientations. J R Soc Interface/R Soc 3(6): 15-35

Giannantoni A, Di Stasi SM, Cucchi A, Mearini E, Bini V, Porena M (2003) Pelvic floor muscle behavior during Valsalva leak point pressure measurement in males and females affected by stress urinary incontinence. J Urol 170(2 Pt 1):485-489

Haworth DJ, Kitta T, Morelli B, Chew DW, Yoshimura N, de Groat WC, Vorp DA (2011) Strain-dependent urethral response. Neurourol Urodyn 30(8):1652-1658 
Holzapfel GA (2000) Biomechanics of soft tissue, the handbook of materials behavior models, vol 3. Academic, Boston, pp 10491063

Holzapfel GA, Gasser TC, Ogden RW (2000) A new constitutive framework for arterial wall mechanics and a comparative study of material models. J Elast Phys Sci Solids 61:1-48

Joller D, Mushkolaj S, Ratia-Garcia J, Marti F, Bachmann A, Müller B (2012) Minipig urethra: a suitable animal model in vitro. Technol Health Care 20(4):329-36

Lalla M, Danielsen CC, Austevoll H, Olsen LH, Jørgensen TM (2007) Biomechanical and biochemical assessment of properties of the anterior urethra after hypospadias repair in a rabbit model. J Urol 177(6):2375-2380

Lecamwasam HS, Sullivan MP, Yalla SV, Cravalho EG (1999) The flow regimes and the pressure-flow relationship in the canine urethra. Neurourol Urodyn 18(5):521-541

Maher E, Creane A, Lally C, Kelly DJ (2012) An anisotropic inelastic constitutive model to describe stress softening and permanent deformation in arterial tissue. J Mech Behav Biomed Mater 12:919

Marti F, Leippold T, John H, Blunschi N, Müller B (2006) Anisotropic Mullins stress softening of a deformed silicone holey plate. Phys Med Biol 51(5):1361

Müller B, Ratia Garcia J, Marti F, Leippold T (2008) Mechanical properties of urethral tissues. J Biomech 41(July):2008

Müller B, Schulz G, Herzen J, Mushkolaj S, Bormann T, Beckmann F, Püschel K (2010) Morphology of urethral tissues. In: Proceedings of SPIE, vol 7804, p 78040D

Natali AN, Carniel EL, Frigo A, Pavan PG, Todros S, Pachera P, Fontanella CG, Rubini A, Cavicchioli L, Avital Y, De Benedictis GM (2016) Experimental investigation of the biomechanics of urethral tissues and structures. Exp Physiol 101(5):641-656

Natali AN, Carniel EL, Fontanella CG, Frigo A, Todros S, Rubini A, De Benedictis GM, Cerruto MA, Artibani W (2017a) Mechanics of the urethral duct: tissue constitutive formulation and structural modeling for the investigation of lumen occlusion. Biomech Model Mechanobiol 16(02):439-447

Natali AN, Carniel EL, Fontanella CG, Todros S, De Benedictis GM, Cerruto MA, Artibani W (2017b) Urethral lumen occlusion by artificial sphincteric devices: a computational biomechanics approach. Biomech Model Mechanobiol 16(4):1-8
Nava A, Mazza E, Kleinermann F, Avis NJ, McClure J, Bajka M (2004) Evaluation of the mechanical properties of human liver and kidney through aspiration experiments. Technol Health Care 12(3):269280

Ophir J, Cespedes I, Ponnekanti H, Yazdi Y, Li X (1991) Elastography: a quantitative method for imaging the elasticity of biological tissues. Ultrason Imaging 13(2):111-134

Peña E, Doblare M (2009) An anisotropic pseudo-elastic approach for modelling Mullins effect in fibrous biological materials. Mech Res Commun 36:784-790

Petiteau JC, Verron E, Othman R, Le Sourne H, Sigrist JF, Barras G (2013) Large strain rate-dependent response of elastomers at different strain rates: convolution integral vs. internal variable formulations. Mech Time Depend Mater 17:349-367

Rebouah M, Chagnon G (2014a) Permanent set and stress-softening constitutive equation applied to rubber-like materials and soft tissues. Acta Mech 225:1685-1698

Rebouah M, Chagnon G (2014b) Extension of classical viscoelastic models in large deformation to anisotropy and stress softening. Int J Nonlinear Mech 61:54-64

Rebouah M, Machado G, Chagnon G, Favier D (2013) Anisotropic Mullins stress softening of a deformed silicone holey plate. Mech Res Commun 49:36-43

Rouiller C (1969) The kidney: morphology, biochemistry, physiology, vol 1. Academic, New York

Samur E, Sedef M, Basdogan C, Avtan L, Duzgun O (2007) A robotic indenter for minimally invasive measurement and characterization of soft tissue response. Med Image Anal 11(4):361-73

Standring S (ed) (2015) Gray's anatomy E-book: the anatomical basis of clinical practice. Elsevier Health Sciences

Tonar Z, Zatura F, Grill R (2004) Surface morphology of kidney, ureters and urinary bladder models based on data from the visible human male. Biomed Pap Med Fac Univ Palacky Olomouc Czech Repub 148(2):249-251

Toungara M, Chagnon G, Geindreau C (2012) Numerical analysis of the wall stress in abdominal aortic aneurysm: influence of the material model near-incompressibility. J Mech, Med Biol 12(01):1250005

Velardo JT (1981) The ureter. Springer, Berlin

Yeoh OH (1993) Some forms of the strain energy function for rubber. Rubber Chem Technol 66(5):754-771 